\title{
Publicidad subliminal en campañas electorales: entre el mito y la realidad
}

\author{
Roberto RODRÍGUEZ ANDRÉS ${ }^{1}$ \\ Universidad Pontificia Comillas ICAI-ICADE \\ Universidad de Navarra
}

Recibido: $12 / 06 / 14$

Aceptado: 10/07/15

\begin{abstract}
Resumen
El interés por la publicidad subliminal ha ocupado un lugar destacado en las últimas décadas en el seno de distintas disciplinas científicas, si bien no termina de haber un consenso acerca de su verdadero poder. Sin embargo, en el campo de la Comunicación Política apenas se encuentran referencias a esta práctica por más que se vengan reportando en los medios desde hace años supuestos casos de anuncios subliminales en campañas electorales. En el presente artículo se propone una aproximación al uso de lo subliminal en la política, para intentar dilucidar si nos encontramos tan sólo ante un mito, como se ha dicho tantas veces al hablar de esta técnica, o por el contrario ante una realidad.
\end{abstract}

Palabras clave: publicidad subliminal, campañas electorales, comunicación política

\section{Subliminal advertising in electoral campaigns: between myth and reality}

\begin{abstract}
During the last decades, the interest in subliminal advertising has been really significant in different scientific disciplines, although there is no consensus about its real influence. However, in the field of Political Communication there are very little references about this practice, even though over recent years there are some reports about cases of subliminal advertisements in electoral campaigns. In this article approach to the use of the subliminal methods in politics is proposed, in order to try to determine whether we are only facing a myth, as it has been said many times regarding this technique, or if we are in front of a reality.

Keywords: subliminal advertising, electoral campaigns, political communication

1 Roberto Rodríguez Andrés es profesor asociado de la Universidad Pontificia Comillas ICAI-ICADE y de la Universidad de Navarra y profesor del Magister de Comunicación y Salud de la Universidad Complutense de Madrid. Director de Comunicación, Marketing y Relaciones Institucionales del Grupo Amma y socio de la firma de asuntos públicos MAS Consulting Group, ha sido Director Adjunto de Comunicación en los Ministerios de Sanidad y de Educación del Gobierno de España y Jefe de Prensa de la Consejería de Educación y Cultura del Gobierno de La Rioja. Ha compatibilizado estos trabajos con la investigación y la docencia universitaria en el campo de la comunicación política, habiendo publicado diversos libros y artículos en revistas científicas. E-mail: rrodrigueza@comillas.edu
\end{abstract}




\section{Introducción}

En el ámbito de la Comunicación Política, uno de los aspectos a los que se ha prestado mayor atención es el de la publicidad, especialmente en campañas electorales. Sin embargo, apenas se encuentran referencias acerca de un tipo concreto de publicidad, la publicidad subliminal, que por el contrario sí ha sido profusamente investigada desde la Psicología y el Marketing.

Podría decirse, en cierto sentido, que la publicidad subliminal es como si no existiera en la comunicación política. Ni en la práctica, porque ningún candidato o partido reconoce haberla usado, ni en la teoría, puesto que este asunto ha concitado escaso interés por parte de los académicos de esta área.

Pero la realidad es que, de forma esporádica, y con mucha repercusión mediática, el debate sobre el posible uso de técnicas subliminales suele plantearse en algunas elecciones, lo que obliga a hacer una reflexión sobre este fenómeno. Una reflexión, además, que recobra actualidad en estos inicios del siglo XXI por cuanto desde distintos ámbitos se empieza de nuevo a hablar del renacimiento de lo subliminal tanto en el mundo comercial como político.

En este contexto, este trabajo se plantea con los siguientes objetivos. En primer lugar, profundizar en si se usa o no la publicidad subliminal en campañas electorales y analizar si esos casos que se vienen reportando desde hace años en los medios son realmente estimulación subliminal u otro tipo de publicidad. En segundo lugar, conocer si estas tácticas tienen o no efectos sobre las actitudes políticas y la conducta de los ciudadanos y, de forma más específica, si son efectivas para influir en su decisión de voto. Y en tercer lugar, ahondar en los aspectos éticos en torno al posible uso de estos anuncios en campañas.

Para tratar de dar respuesta a estos objetivos, el presente trabajo se basará fundamentalmente en una revisión bibliográfica de las aproximaciones que se han realizado desde mediados del siglo XX sobre este fenómeno tanto en el campo de la publicidad y el marketing como en el de la psicología y también, aunque de forma más incipiente, en el de la comunicación política. Esta revisión se centrará tanto en libros académicos y artículos de investigación como también en obras de carácter más divulgativo, sondeos y cuestionarios a profesionales y ciudadanos e informaciones periodísticas, habida cuenta de que este fenómeno, como hemos apuntado, ha concitado el interés mediático y también de la opinión pública durante las últimas décadas, lo que lo ha convertido en un área no sólo de investigación científica sino también de interés popular.

\section{Aproximación al concepto y a los orígenes de la estimulación subliminal}

Según el diccionario de la Real Academia, subliminal es aquello «que está por debajo del umbral de la conciencia» o «que por su debilidad o brevedad no es percibido conscientemente, pero influye en la conducta». Esta noción es la que se extrae también 
del examen etimológico del término así como de las definiciones académicas que se han propuesto, que la califican como aquellos estímulos visuales o auditivos que llegan al cerebro humano sin que éste sea consciente de ello pero logrando influir en la conducta de la persona ${ }^{2}$. Es común en estas definiciones, por tanto, el presuponer no sólo la existencia de estos estímulos y la capacidad del ser humano para captarlos, sino también su supuesta eficacia a la hora de condicionar el comportamiento.

El interés por lo subliminal procede de antiguo. Como recuerda Key, «la existencia de la inconsciencia humana ha sido documentada a través de muchos siglos» ${ }^{3}$. Según este autor, la primera mención fue realizada en el año 400 antes de Cristo por Demócrito y sería desarrollada posteriormente por Platón. También Aristóteles se referiría a la posibilidad de una percepción subliminal, al igual que siglos más tarde Montaigne y Leibniz, quien llegó a afirmar que sería «un gran error creer que no hay más percepción en el alma que aquélla de la que se es consciente» ${ }^{4}$.

Sin embargo, la mayor parte de las investigaciones se han producido en fechas más recientes, principalmente a partir de finales del siglo XIX. De esta época son las teorías de Freud sobre el subconsciente, que serían la base de los trabajos de uno de sus colaboradores, el doctor Poetzl, que estimó que de las 100.000 fijaciones que hacen los ojos a lo largo del día, sólo una pequeña cantidad es captada conscientemente, mientras que el resto llega a nuestro cerebro sin que nos percatemos de ello ${ }^{5}$.

Estos primeros avances en la investigación vendrían acompañados de una larga lista de nuevos estudios, sobre todo en el ámbito de la Psicología. Como indica Pinillos, esta generalización se produciría en Estados Unidos a partir de la década de los treinta y, con más o menos intensidad, ha continuado hasta nuestros días 6 .

\section{La publicidad subliminal}

Para la opinión pública, sin embargo, la estimulación subliminal no sería conocida hasta que empezó a aplicarse en el campo de la publicidad. Sutil apunta que algunos publicitarios como Hollingworth en 1913 y Cheskin en 1948 ya mencionaron la

2 García Matilla, E. (1979): «Nuevas técnicas de manipular», Mensaje y Medios, 6, enero, 41-45; Moore, T.e. (1982): «Subliminal advertising: what you see is what you get», Journal of Marketing, 46 (2), 38-47; BornsteIn, R.F. (1989): «Subliminal techniques as propaganda tools: review and critique», The Journal of Mind and Behavior, 10 (3), 231-262; DurÁn, A. (1989): Psicología de la publicidad y de la venta, Barcelona: CeAc; Lillo, J. (1993): Psicología de la percepción, Madrid: Debate; González, J.L. (1994): Persuasión subliminal y sus técnicas, Madrid: Biblioteca Nueva; Becerra Navarro, A.B. (2005): «El mensaje subliminal: tácticas de publicidad ilícita», Información para la paz, Valencia: Fundación COSO, 169-182.

3 Key, W.B. (1991): Seducción subliminal, Buenos Aires: Vergara, 45.

4 Brown, J.A.c. (1986): Técnicas de persuasión: de la propaganda al lavado de cerebro, Madrid: Alianza.

5 Poetzl, O. (1917): «The relationship between experimentally induced dream images and indirect vision», Psychological Issues, 2, 41-120.

6 Pinillos, J.t. (1995): Principios de psicología, Madrid: Alianza. 
posibilidad de aplicar estas técnicas al ámbito publicitario ${ }^{7}$. No obstante, García Matilla concreta que la primera referencia sobre el empleo de estos estímulos en publicidad se encontró el 10 de junio de 1956 en el London Sunday Times. En un artículo titulado «Ventas a través del subconsciente», se hacía mención a los experimentos subliminales que diversos investigadores habían llevado a cabo en Estados Unidos para aumentar la venta de helados ${ }^{8}$.

Pero la publicidad subliminal adquiriría verdadera relevancia pública de la mano de James Vicary, investigador de mercado que ha pasado a la historia como el precursor de esta práctica gracias al experimento que llevó a cabo en 1957 en un cine de Nueva Jersey en el que intercaló mensajes subliminales como «Beba Coca-Cola» y «Coma palomitas» y que, según afirmó, sirvieron para aumentar significativamente las ventas de ambos productos 9 .

Ese mismo año, el debate sobre este tipo de publicidad se avivó aún más con la publicación del libro The Hidden Persuaders, de Vance Packard, en el que se detallaban las estrategias subliminales a las que recurrían los comerciantes para conseguir captar clientes ${ }^{10}$. Y todo ello en una época en la que el auge económico desencadenó una fiebre consumista y provocó una feroz competencia en el mercado, lo que trajo consigo la experimentación de nuevas vías para llamar la atención de los consumidores y la contratación de psicólogos para conocer los resortes de la motivación ${ }^{11}$.

El interés por el tema fue decayendo en los años sesenta, pero en la década siguiente tres autores volvieron a poner de actualidad la publicidad subliminal. El primero de ellos fue Hawkins, que tras llevar a cabo diversos experimentos empíricos dio por probada su efectividad ${ }^{12}$. Un año después, el segundo de los autores, Dixon, publicó la obra Percepción subliminal, que contribuyó a la divulgación de los avances científicos que se habían ido planteando sobre esta materia ${ }^{13}$. Finalmente, el profesor norteamericano Wilson Bryan Key irrumpió en este debate en 1973 con el libro Seducción subliminal, que gozó de gran popularidad y sirvió para aumentar el mito que se había creado alrededor

7 Sutil, L. (1995): Estimulación subliminal: mensajes ocultos que dirigen nuestra vida, Madrid: Vergara.

8 García Matilla, E. (1979): «Nuevas técnicas de manipular», Mensaje y Medios, 6, enero, 41-45.

9 Gold, P. (1987): Advertising, politics, and American culture: from salesmanship to therapy, Nueva York: Paragon House.

10 Packard, V. (1961): Las formas ocultas de la propaganda, Buenos Aires: Editorial Sudamericana.

11 Phillips, M.J. (1997): Ethics and manipulation in advertising, Westport: Quorum Books; SAmuel, L.R. (2013): Freud on Madison Avenue: motivation research and subliminal advertising in America, Philadelphia: University of Pennsylvania Press.

12 Hawkins, D. (1970): "The effects of subliminal stimulation on drive level and brand preference», Journal of Marketing Research, 7, agosto, 322-326.

13 Dixon, N.F. (1971): Subliminal perception: the nature of a controversy, Londres: McGraw-Hill. 
de este fenómeno ${ }^{14}$. En esta obra, que tendría su continuación con varios trabajos más, Key proponía numerosos ejemplos de publicidad de todo tipo de productos y servicios con estímulos camuflados, la mayoría de ellos de naturaleza sexual.

Pronto se provocó un gran revuelo entre la opinión pública norteamericana. Además, no hay que olvidar que los estadounidenses habían tenido noticia, también a partir de los años 50, del llamado «lavado de cerebro» en la Guerra de Corea, lo que propició que la población viviera una especie de «psicosis» en torno a la posible utilización de todos estos métodos de control ${ }^{15}$.

Ante esta situación, comenzó a generarse preocupación por regular este fenómeno e incluso se planteó la conveniencia de prohibirlo. Uno de los primeros organismos en posicionarse fue el British Institute of Practitioners in Advertising, que en 1958 alertó a sus asociados de que no usaran este tipo de publicidad ${ }^{16}$. Restricciones similares fueron implantándose en otros países ${ }^{17}$ e incluso varios organismos internacionales como la ONU y la Unión Europea recomendaron abiertamente su prohibición ${ }^{18}$.

En cuanto a España, tanto el conocimiento de este fenómeno como la incorporación del término subliminal al lenguaje coloquial se produjeron en fechas más recientes. De hecho, el diccionario de la Real Academia no lo mencionó hasta su vigésima edición (1984). Sin embargo, ya desde años antes hubo voces en nuestro país que empezaron a alertar de la necesidad de regularla, como Muñoz Espinalt ${ }^{19}$ y García Matilla ${ }^{20}$, antes de que quedara definitivamente prohibida por la Ley General de Publicidad de 1988, al considerarla ilícita ${ }^{21}$.

En Estados Unidos, por el contrario, los intentos de regulación se toparon con dificultades. Poco después de los experimentos de Vicary se pusieron en marcha varias iniciativas con este propósito, pero prácticamente ninguna tuvo éxito ${ }^{22}$. No obstante, y

\footnotetext{
14 Key, W.B. (1991): Seducción subliminal, Buenos Aires: Vergara.

15 Ibidem.

16 Brown, J.A.c. (1986): Técnicas de persuasión: de la propaganda al lavado de cerebro, Madrid: Alianza.

17 Durandin, G. (1983): La mentira en la propaganda política y en la publicidad, Barcelona: Paidós.

18 García Matilla, E. (1979): «Nuevas técnicas de manipular», Mensaje y Medios, 6, enero, 41-45; GonzÁlez, J.L. (1994): Persuasión subliminal y sus técnicas, Madrid: Biblioteca Nueva.

19 Muñoz Espinalt, C. (1963): Psicología de la publicidad, Barcelona: Toray.

20 García Matilla, E. (1979): «Nuevas técnicas de manipular», Mensaje y Medios, 6, enero, 41-45.

21 Méndez, R.M. y Vilalta, A.e. (1999): La publicidad ilícita: engañosa, desleal, subliminal y otras, Barcelona: Bosch.

22 Edmonds, T.N. (1988): «Regulate political ads?», Advertising Age, 59 (51), noviembre, 17-20; PhILliPs, M.J. (1997): Ethics and manipulation in advertising, Westport: Quorum Books.
} 
aún no estando prohibida, está ampliamente generalizado el rechazo a esta práctica por ser una ofensa contra el interés público ${ }^{23}$.

Pero antes de concluir este apartado sobre la publicidad subliminal, conviene precisar que la mayor parte de las veces se han calificado como subliminales anuncios que en realidad no lo son, o por lo menos no en el sentido literal. Surge aquí la diferenciación entre percepción subliminal y percepción inconsciente. Como indica González, «aunque toda percepción subliminal es inconsciente, no toda percepción inconsciente es subliminal. Por ejemplo, una percepción inconsciente por falta de atención no se debe a que el estímulo esté por debajo del umbral, sino a que no es advertido» ${ }^{24}$.

Es éste el caso de los estímulos ocultos o enmascarados, de los mensajes o imágenes que juegan con la ambigüedad o de la publicidad encubierta, que presenta solapadamente productos en el transcurso de espacios televisivos o cinematográficos. En ocasiones este tipo de publicidad ha sido catalogada como subliminal, puesto que intenta influir en el ciudadano de forma velada, sin que el producto ocupe el centro de atención pero captando su interés de forma indirecta.

Sin embargo, esto no sería propiamente publicidad subliminal. En un sentido estricto, este término debería emplearse sólo para aquellos estímulos visuales o sonoros de una breve fracción de segundo de duración, emitidos por aparatos especializados, que consiguen posicionarse en niveles fronterizos al umbral de la conciencia de tal forma que, aunque seas advertido, no seas capaz de apreciarlos conscientemente pero sí a través de tu subconsciente. No es el caso de la publicidad encubierta, en la que los estímulos pueden ser percibidos afinando la vista, el oído o la atención ${ }^{25}$. Lo que ocurre es que, como apunta Núñez, en la publicidad se trabaja con un concepto de subliminal más amplio y flexible que en el campo de la psicología ${ }^{26}$. Y esto mismo es lo que lleva a Ferrés a afirmar que «en un sentido más amplio se considera subliminal cualquier estímulo que no es percibido de manera consciente, por el motivo que sea» ${ }^{27}$.

23 Silverglate, S. (1990): «Subliminal perception and the first amendment: yelling fire in a crowded mind?», University of Miami Law Review, 44, 1243-1281.

24 GonzÁlez, J.L. (1994): Persuasión subliminal y sus técnicas, Madrid: Biblioteca Nueva, 52.

25 Dixon, N.f. (1971): Subliminal perception: the nature of a controversy, Londres: McGrawHill; Moore, T.E. (1982): «Subliminal advertising: what you see is what you get», Journal of Marketing, 46 (2), 38-47; García Matilla, E. (1990): Subliminal: escrito en nuestro cerebro, Madrid: Bitácora; Méndez, R.m. y Vilalta, A.e. (1999): La publicidad ilícita: engañosa, desleal, subliminal y otras, Barcelona: Bosch; Perloff, R.M. (2010): The dynamics of persuasion: communication and attitudes in the 21st. century, Nueva York: Routledge.

26 NúÑez Partido, J.P. (2000): «Publicidad subliminal. Mito y realidad», Rev. Razón y Fe, 1223-1224, 207-219.

27 Ferrés, J. (1996): Televisión subliminal, Barcelona: Paidós, 16. 


\section{La publicidad subliminal en comunicación política y campañas electorales}

Pero el mito de la publicidad subliminal no se quedó en el ámbito comercial y pronto empezó a hablarse de su uso por parte de los políticos. De hecho, cuenta García Matilla que ya Goebbels habría recurrido a ella en la Alemania nazi ${ }^{28}$.

No obstante, fue el conocimiento de los experimentos de Vicary, recogidos con profusión en los medios, y la publicación del libro de Packard los que contribuyeron a levantar una gran polémica sobre el posible uso de la publicidad subliminal por parte de los políticos. Y es que, como escribió Cousins, editor del The Saturday Review, «si el invento vale para las palomitas de maíz, ¿por qué no para los políticos? $\gg^{29}$.

A inicios de 1958, la revista Life se sumó a la controversia, al dedicar un amplio reportaje a las técnicas subliminales en el que afirmaba que estaban aplicándose ya en campañas electorales. Ese mismo año, el escritor británico Aldous Huxley sugirió en una entrevista televisiva que la técnica utilizada por Vicary había mostrado su eficacia y que para mediados de los sesenta sería posible conquistar el voto sin que los ciudadanos se percataran siquiera de que estaban siendo influidos ${ }^{30}$. Y unos años después, en 1973, Key afirmaría que el gobierno de Estados Unidos y los partidos políticos norteamericanos estaban haciendo ya un uso indiscriminado de todo tipo de mensajes subliminales durante las campañas y que la propia CIA los utilizaba contra los soviéticos ${ }^{31}$.

Poco a poco, como había ocurrido en el ámbito comercial, la polémica se fue mitigando. Pero en el año 2000 volvió a irrumpir con gran estruendo, cuando la prensa mundial se hizo eco de la supuesta utilización de estímulos subliminales en uno de los spots producidos por el Comité Nacional Republicano en favor del candidato a la Casa Blanca George W. Bush. Al analizar el spot fotograma a fotograma, se descubrió que aparecía la palabra «RATS» (ratas) cuando se hacían alusiones al candidato demócrata Albert Gore y a su propuesta de reforma sanitaria ${ }^{32}$.

Desde el equipo de Gore se lanzaron fuertes críticas, aduciendo a que «nunca habíamos visto nada como esto». Sin embargo, el propio Bush negó el uso de estímulos subliminales, llegando incluso a declarar que todo aquello era «ridículo». Lo mismo repitieron tanto su jefe de publicidad, Mark McKinnon, como el creador del anuncio, el veterano consultor republicano Alex Castellanos. «Es una acusación muy tonta la que están haciéndome; no soy tan inteligente», afirmó a los medios, al tiempo que

\footnotetext{
28 García Matilla, E. (1979): «Nuevas técnicas de manipular», Mensaje y Medios, 6, enero, 41-45.

29 Cousins, N. (1957): «Smudging the subconscious», Saturday Review, 40, 5 de octubre, 20.

30 Brown, J.A.c. (1986): Técnicas de persuasión: de la propaganda al lavado de cerebro, Madrid: Alianza.

31 KeY, W.B. (1991): Seducción subliminal, Buenos Aires: Vergara.

32 MARK, D. (2006): Going dirty: the art of negative campaigning, Lanham: Rowman \& Littlefield Publishers, Inc.
} 
se defendía aludiendo a que no había en el anuncio ninguna intención de manipular psicológicamente a los espectadores. Según Castellanos, se trataba de una simple coincidencia, ya que las letras que se usaron para que pasaran fugazmente por la pantalla no eran más que un fragmento de la palabra «burocrats». «Es un truco visual. La gente se aburre viendo la tele y uno trata de involucrarla».

El anuncio fue emitido un par de semanas antes de que fuera retirado (según el equipo de Bush porque estaba programado así de antemano, no por la polémica), pero se pudo ver en numerosos Estados en un momento decisivo de la campaña. Y aunque el Partido Demócrata denunció el asunto ante la Federal Communications Commission (FCC), ésta concluyó su investigación bastantes meses después sin aplicar ninguna sanción ${ }^{33}$.

Según la Asociación Americana de Consultores Políticos, era la primera vez que se usaban técnicas subliminales en una campaña presidencial, una opinión compartida por el investigador Darrell West en diversas entrevistas que se le realizaron durante esos días. Sin embargo, según Jamieson ${ }^{34}$, hubo un antecedente, aunque es verdad que no en una carrera por la presidencia. El anterior fue diseñado también por Alex Castellanos en 1990 en la campaña del candidato republicano para el Senado por Carolina del Norte, Jesse Helms, en el que se colaron imágenes racistas contra el rival demócrata, el empresario de color Harvey Gant. Y también casualmente, Castellanos se defendió entonces de las acusaciones diciendo, como repitió en el 2000, que todo era meramente accidental.

Después de «RATS» ha habido otros casos en los que se ha denunciado el uso de estímulos subliminales, todos ellos en spots negativos, como ocurrió en la campaña presidencial de 2008. Primero fue un spot de Mitt Romney contra John McCain en las primarias republicanas, en el que volvía a aparecer destacada la palabra «RAT» cuando se hacía mención a McCain. De nuevo se volvió a hablar de Alex Castellanos, que era consultor de Romney, como el creador de este anuncio ${ }^{35}$. Y poco después, en esa misma campaña, el ya candidato republicano John McCain produjo un spot bajo el título de «The Obama Iraq Documentary» en el que se volvió a denunciar el uso de técnicas subliminales. Al inicio del anuncio, las letras del título iban apareciendo de forma gradual y en un momento se detenían por una breve fracción de segundo en unas siglas que muchos asociaron con Al Qaeda («al q D»). Puede parecer casual, como adujeron desde el equipo de McCain, pero hay que recordar que la asociación de Obama con el

\footnotetext{
33 Acland, C.R. (2011): Swift viewing: the popular life of subliminal influence, Durham, NC: Duke University Press.

34 Jamieson, K.H. (1992): Dirty politics, Nueva York: Oxford University Press.

35 Stein, S. (2008): «Does Romney's ad against McCain contain a subliminal message?». En Huffington Post, 28 de marzo, http://www.huffingtonpost.com/2008/01/04/does-romneys-adagainst-m_n_79905.html Web visitada el 15/02/2014.
} 
islam, incluso con el terrorismo, fue un argumento utilizado por los republicanos en esa campaña ${ }^{36}$. Y aunque no se confirmó oficialmente, se dijo que detrás de ese spot estaba de nuevo la mano de Castellanos ${ }^{37}$.

Avanzando en el tiempo, el último ejemplo en Estados Unidos se produjo en julio de 2011, cuando la organización conservadora American Crossroads, creada por el gurú electoral de George W. Bush, Karl Rove, emitió un anuncio en Internet contra Obama, en el que cada vez que aparecía la imagen del presidente se sobreimpresionaba la palabra «Taxes» («Impuestos») de forma subliminal ${ }^{38}$.

Pero Estados Unidos no ha sido el único país en el que se han reportado supuestos casos de este tipo de publicidad. Por ejemplo, en la campaña presidencial venezolana de 1998, uno de los spots de la candidata Irene Sáez incluía por dos veces el mensaje sobreimpresionado «Vota Irene» de forma subliminal ${ }^{39}$. Y también se acusó al mexicano Felipe Calderón de usar estos estímulos en un anuncio contra López Obrador en la campaña presidencial de $2006^{40}$.

En cualquier caso, las noticias sobre este asunto han sido más frecuentes en aquellos países en los que no existe ninguna traba legal a la emisión de spots durante las campañas y en los que, por tanto, la publicidad política tiene mayor relevancia e impacto sobre la opinión pública. En los últimos años, además, está aumentando el número de casos en anuncios concebidos exclusivamente para su difusión a través de Internet, que en ocasiones consiguen saltarse las posibles barreras legales en los países de origen mediante el uso de servidores que están alojados en países terceros donde no hay prohibición de estas técnicas.

En cuanto al caso español, ha habido igualmente varios ejemplos de acusaciones entre partidos por supuestos usos de publicidad subliminal en campañas electorales ${ }^{41}$, si bien es verdad que todos ellos han sido mensajes encubiertos más que propiamente subliminales. La primera polémica surgió en la campaña de 1986, durante la retransmisión

\footnotetext{
36 Acland, C.R. (2011): Swift viewing: the popular life of subliminal influence, Durham, NC: Duke University Press.

37 Belle, N. (2008): «Subliminal message in McCain's new ad?». En Crooks and Liars, 19 de julio, http://crooksandliars.com/2008/07/20/subliminal-message-in-mccains-new-ad Web visitada el 15/02/2014.

38 PeytiBI, X. (2011): «La mano de Karl Rove es alargada», en el blog Xabier Peytibi, Comunicación política, campañas, redes, 8 de julio, http://www.xavierpeytibi.com/2011/07/08/ la-mano-karl-rove-alargada/ Web visitada el 14/03/2014.

39 Morao, J. (2011): «¿Es efectiva la publicidad subliminal?». En Jingle Electoral, 2 de septiembre, http://jingleelectoral.com/2011/09/02/de-la-llamada-publicidad-subliminal/ Web visitada el 14/02/2014.

40 ACland, C.R. (2011): Swift viewing: the popular life of subliminal influence, Durham, NC: Duke University Press.

41 Rivera Camino, J. y Sutil Martín, L. (2004): Marketing y publicidad subliminal, Madrid: ESIC Editorial.
} 
del partido de fútbol del Mundial de México entre Dinamarca y España. Cuando se emitía la repetición de uno de los goles de la selección, apareció un rótulo con las letras «PSOE» en vez del nombre del jugador que había marcado el gol. Aunque TVE se defendió alegando un fallo en la máquina rotuladora, periodistas y políticos hablaron de que ese rótulo era un claro ejemplo de estimulación subliminal, puesto que pretendía identificar la alegría del gol con las siglas del partido de gobierno ${ }^{42}$.

A partir de entonces, PSOE y PP se han lanzado reproches por utilizar técnicas subliminales en campaña. En 1989, por ejemplo, se atacó al PSOE por un spot en el que aparecían imágenes a gran velocidad con rostros de personajes conocidos de la vida cultural y social del país y, en 1996, por el vídeo del dóberman. Por su parte, los populares fueron acusados en las elecciones generales de 2000 y en las municipales y autonómicas de 2003 de utilizar en sus anuncios el mismo fondo cromático y tipografía que los de los informativos de TVE.

Los dos últimos casos hasta el momento se han dado en 2006 y 2011. En el primero, el PP denunció que el Gobierno había insertado subliminalmente propaganda del PSOE en un anuncio institucional de ADIF en el que, según los populares, aparecían dos jóvenes con carteles electorales en la mano, uno pidiendo el voto para un candidato muy similar a Felipe González y otro con la imagen de otro muy parecido al presidente Zapatero. Y ya en 2011, el PP criticó que TVE estaba haciendo publicidad subliminal en favor del PSOE. Durante la emisión de uno de los programas del Canal 24 Horas, el PP alertó de que había un monitor detrás de la presentadora en el que se pudo ver durante varios minutos el eslogan «Rubalcaba sí» e imágenes del candidato socialista. TVE se defendió aludiendo a que ese monitor reproducía la señal de La 1, en la que en ese momento se emitían los espacios gratuitos de publicidad electoral y que se pudo ver tanto el del PSOE como los de otros partidos.

\section{La opinión de ciudadanos, publicitarios y consultores políticos sobre la publicidad subliminal}

Con el paso de los años y la publicación de supuestos casos de publicidad subliminal, tanto en el ámbito comercial como político, la opinión pública ha ido conociendo esta técnica y, al mismo tiempo, ha ido confiando cada vez más en su supuesto poder. En 1959, tan sólo dos años después de los experimentos de Vicary, un sondeo realizado en Estados Unidos mostró que el $41 \%$ de los encuestados había oído hablar ya de la publicidad subliminal ${ }^{43}$, lo que da buena muestra de la enorme relevancia que adquirió este fenómeno. Veinticuatro años después, en 1983, otra encuesta elevaba este porcentaje hasta el $81 \%^{44}$ y en 1994 , otro sondeo lo situaba en el $75 \%{ }^{45}$. En cuanto al

42 Hermida, J.m. (1993): La estrategia de la mentira, Madrid: Temas de Hoy.

43 Haber, R.n. (1959): «Public attitudes regarding subliminal advertising», Public Opinion Quarterly, 23, verano, 291-293.

44 Zanot, E.J.; Pincus, J.D. y LAmp, E.J. (1983): «Public perceptions of subliminal advertising», Journal of Advertising, 12 (1), 39-45.

45 ACland, C.R. (2011): Swift viewing: the popular life of subliminal influence, Durham, NC: Duke University Press. 
caso español, una encuesta de 1995 constataba también que el 80\% reconocía haber oído hablar alguna vez de la publicidad subliminal, un $84 \%$ creía que los publicitarios recurren a estos mensajes de forma habitual y un $72 \%$ que estas técnicas son eficaces ${ }^{46}$.

Esta creencia, aunque con matices, es también compartida por los propios publicitarios. En un análisis llevado a cabo en 1994 en Estados Unidos se reveló que el $66 \%$ de ellos manifestaba que las técnicas subliminales se estaban empleando con frecuencia, aunque algo más del $50 \%$ creía que no eran efectivas ${ }^{47}$. Sin embargo, algunos destacados profesionales han puesto en duda tanto su uso como su supuesto poder. Dice O'Toole que «no hay tal cosa de la publicidad subliminal. Yo jamás he visto un ejemplo de ella, ni jamás he oído una discusión seria sobre esta técnica entre los que se dedican a la publicidad $»^{48}$. Por su parte, Ogilvy cree que «ningún publicitario ha usado jamás la publicidad subliminal $\rangle^{49}$.

Pero a pesar de estas afirmaciones, Haberstroh (1994) cree que buena parte de la culpa de que la opinión pública haya seguido creyendo en el uso de la publicidad subliminal la han tenido los propios publicitarios, que durante muchos años, mientras personas como Packard y Key difundían sus teorías, no salieron públicamente a negar este uso. Hicieron del silencio su única estrategia de respuesta, lo que contribuyó a aumentar las dudas. Y también el mito.

En el entorno de la consultoría política resulta más complicado encontrar manifestaciones en torno a este asunto, más allá de las que se produjeron tras la polémica del spot de «RATS». Ante esta falta de referencias, para el presente artículo se ha pedido la opinión de alguno de los principales consultores políticos españoles, con experiencia también en campañas internacionales: José Luis Sanchís, Presidente de Sanchís\&Asociados, Presidente de Honor de Torres y Carrera y único español en haber sido Presidente de la Asociación Internacional de Consultores Políticos (1980-1982); Daniel Ureña, Socio-Director General de MAS Consulting Group y Vicepresidente de la Asociación Europea de Consultores Políticos; Antoni Gutiérrez-Rubí, Director de Ideograma; Luis Arroyo, Presidente de Asesores de Comunicación Pública; Antonio Núñez, Global Brand Strategic Planner en Young\&Rubicam Group y Marcos Magaña, General Manager for Latin America de Arista.

A la pregunta de si se ha empleado en alguna ocasión la publicidad subliminal en política, ninguno lo niega. Como afirma Núñez, los primeros experimentos de Vicary y las experiencias que vinieron después «no creo que pasaran desapercibidas para los

\footnotetext{
46 Merino, D. (1995): Publicidad subliminal y eficacia publicitaria, Madrid: Comunidad de Madrid.

47 Rogers, M. y SeILER, C.A. (1994): «The answer is no: a national survey of advertising industry practitioners and their clients about whether they use subliminal advertising», Journal of Advertising Research, 34 (2), 36-45.

48 O'toole, J. (1985): The trouble with advertising, Nueva York: Times Books, 16.

49 Ogilvy, D. (1985): Ogilvy on advertising, Nueva York: Vintage Books, 209.
} 
profesionales de la comunicación política, así que veo factible que se haya investigado y probado esta técnica en campañas electorales». Este consultor sugiere, de hecho, que en el contexto de la Segunda Guerra Mundial, tanto americanos como británicos pudieron ya utilizar estímulos subliminales en su propaganda, con el objetivo de «lograr la rendición del enemigo o elevar la moral de la ciudadanía en una situación desesperada».

Sin embargo, todos coinciden en que ellos nunca han usado publicidad subliminal, que ningún candidato les ha pedido utilizarla y que no conocen ningún caso en primera persona en el que esto haya ocurrido. Según Sanchís, «no he visto nunca un anuncio así en 37 años de profesión; la publicidad subliminal está prohibida, es casi imposible hacerla y además puede provocarte graves problemas». «El uso de esta publicidad es sumamente esporádico -dice Arroyo-. Yo no me he encontrado nunca a un candidato, una campaña o un creativo publicitario que pidiera aplicar estímulos subliminales». Magaña, por su parte, afirma igualmente que «nunca me he topado con ningún caso real de aplicación en campaña electoral. Ningún partido o cliente lo ha pedido y yo no lo he sugerido». Lo mismo repite Ureña, que habla también de una especie de «leyenda negra» sobre esta cuestión, por la mala imagen que tiene este tipo de publicidad, lo que hace que sea prácticamente imposible encontrar algún consultor o candidato que reconozca haberla usado. Y Núñez concluye en esta misma línea afirmando que «cualquier comunicador negaría en rotundo el estudio de mensajes subliminales, por las connotaciones negativas del término, manipulación, engaño, etc.».

\section{La controversia sobre la efectividad de las técnicas subliminales}

En el debate sobre la publicidad subliminal la polémica no se centra sólo en saber si se está usando o no. Otro de los aspectos que más controversia ha suscitado es el de su supuesta eficacia, es decir, comprobar hasta qué punto es real ese poder que se le confiere por parte de la opinión pública. Y este aspecto, en el campo concreto de la Comunicación Política, gira en torno a los efectos que pueden tener estos anuncios sobre la intención de voto. Pero para ello, es necesario adentrarse en primer lugar en las investigaciones que se han llevado a cabo hasta el momento en campos como la Psicología y el Marketing, puesto que han servido para poner las bases de los estudios aplicados a la política. 


\subsection{Los ensayos en el ámbito de la Psicología}

Son numerosas las investigaciones que concluyen que el ser humano es capaz de captar estímulos subliminales de forma inconsciente ${ }^{50}$. Como afirma García Matilla,

estas aproximaciones «han demostrado científicamente que los estímulos subliminales pueden llegar a nuestro cerebro. Pero el paso siguiente es conocer qué efectos pueden producir estos mensajes en el comportamiento del ser humano» ${ }^{51}$.

A este respecto, González llevó a cabo a inicios de los 90 un análisis de los experimentos realizados en este terreno, que le permitió afirmar que «los estímulos visuales y auditivos, a nivel subliminal, tienen efectos constatables en multitud de comportamientos del individuo (...). En general, puede mantenerse que la estimulación subliminal puede introducir cambios en todo tipo de conductas $\rangle^{52}$.

Este tipo de investigaciones, aunque se han extendido a numerosos ámbitos, se han realizado sobre todo desde la psicología básica, aunque también se han producido avances en la psicología aplicada, en donde se ha apuntado que esta técnica podría tener consecuencias terapéuticas en ciertos trastornos como depresión, obesidad o adicción a las drogas ${ }^{53}$.

En cualquier caso, en este debate no se debe olvidar que la idea de subliminalidad depende en gran medida del sujeto receptor. El denominado «umbral de la conciencia» no es igual para todos los seres humanos e, incluso, tampoco es siempre el mismo para la misma persona ${ }^{54}$. En palabras de Moore, dicho umbral puede variar no sólo de una persona a otra, sino en un mismo individuo de una situación a otra, de un día a

50 Bevan, W. (1964): «Subliminal stimulation: a pervasive problem for psychology», Psychological Bulletin, 61 (2), 89-99; SAEGERT, J. (1979): «Another look at subliminal perception», Journal of Advertising Research, 19 (1), 55-57; Moore, T.E. (1982): «Subliminal advertising: what you see is what you get», Journal of Marketing, 46 (2), 38-47; CuPERFAIN, R. y Clarke, T.K. (1985): «A new perspective of subliminal perception», Journal of Advertising, 14 (1), 36-41; BoRnsteIn, R.F. (1989): «Subliminal techniques as propaganda tools: review and critique», The Journal of Mind and Behavior, 10 (3), 231-262; BEATTY, S.E. y Hawkins, D.I. (1989): «Subliminal stimulation: some new data and interpretation», Journal of Advertising, 18 (3), 4-8; MoIx, J. (1997): «Percepción subliminal de estímulos de corta duración», Psicologemas, $11(22), 189-208$.

51 García Matilla, E. (1990): Subliminal: escrito en nuestro cerebro, Madrid: Bitácora, 168.

52 GonzÁlez, J.L. (1994): Persuasión subliminal y sus técnicas, Madrid: Biblioteca Nueva, 117.

53 Merino, D. (1995): Publicidad subliminal y eficacia publicitaria, Madrid: Comunidad de Madrid; Sutı, L. (1995): Estimulación subliminal: mensajes ocultos que dirigen nuestra vida, Madrid: Vergara.

54 Durán, A. (1989): Psicología de la publicidad y de la venta, Barcelona: CEAC; SUTIL, L. (1995): Estimulación subliminal: mensajes ocultos que dirigen nuestra vida, Madrid: Vergara. 
otro o, incluso, de un minuto a otro ${ }^{55}$. A este respecto, González precisa que «multitud de experiencias de laboratorio demuestran que la tensión psicológica, la angustia, las emociones y la excitación hacen variar el umbral de reconocimiento (...). No existe, pues, un umbral sensorial fijo, sino que depende, en sus variaciones, de condiciones psíquicas, en general, y de factores cognitivos y emocionales en especial» ${ }^{56}$.

\subsection{Investigaciones sobre el poder de la estimulación subliminal en formas publicitarias}

Pero ¿qué ocurre cuando estos estímulos subliminales traspasan las barreras del laboratorio y se aplican en la vida real? ¿Son igualmente eficaces en el ámbito publicitario? Los mismos investigadores que han analizado los efectos en el campo de la psicología han limitado su alcance, aludiendo, como apunta Merino, a que «el hecho de que en estos estudios se trabaje con poblaciones patológicas, o el que los sujetos estén predispuestos a cambiar su conducta, imposibilitan la generalización de resultados a otras áreas aplicadas $\iota^{57}$. En definitiva, como apunta Perloff, «es importante distinguir entre la percepción subliminal en la teoría y la percepción subliminal en la realidad de la publicidad $\rangle^{58}$.

Algunos van más allá, insinuando que fuera del laboratorio no sólo es difícil encontrar secuelas de la estimulación subliminal sino que incluso lo es aún más el tratar de demostrar que esos estímulos lleguen a ser percibidos por el ser humano. Así lo entiende Clark, que afirma que «sigue en pie la controversia relativa a la existencia de una percepción subliminal en condiciones normales no controladas. Fuera del laboratorio, el individuo se ve forzado a seleccionar entre numerosos estímulos que concurren por encima del nivel sensorial absoluto. ¿Es posible en esas condiciones que reaccione a un estímulo que se le presente por debajo de dicho nivel? $\rangle^{59}$.

En definitiva, ¿hasta qué punto es eficaz un anuncio con apelaciones subliminales cuando el espectador tiene a su alrededor cientos de elementos de distracción que pueden desviar su capacidad de permanecer atento? Como afirma Cavazza, es bastante complicado admitir que en estas circunstancias los mensajes subliminales puedan tener los efectos que se le han concedido ${ }^{60}$.

Y, de hecho, esto es lo primero que la comunidad investigadora quiso comprobar en torno a los experimentos de Vicary, a los que desde el inicio concedieron escasa credibilidad. Se le pidieron informes y pruebas, pero no los pudo ofrecer. Ante los numerosos ataques que empezó a recibir, el mismo Vicary intentó suavizar sus

55 Moore, T.E. (1982): «Subliminal advertising: what you see is what you get», Journal of Marketing, 46 (2), 38-47.

56 GonzÁlez, J.L. (1994): Persuasión subliminal y sus técnicas, Madrid: Biblioteca Nueva, 52-54.

57 Merino, D. (1995): Publicidad subliminal y eficacia publicitaria, Madrid: Comunidad de Madrid, 5.

58 Perloff, R.M. (2010): The dynamics of persuasion: communication and attitudes in the 21st. century, Nueva York: Routledge, 290.

59 Clark, E. (1989): La publicidad y su poder, Barcelona: Planeta, 156.

60 Cavazza, N. (1999): Comunicación y persuasión, Madrid: Acento. 
conclusiones iniciales. Incluso se vio forzado a repetir el experimento bajo supervisión externa, esta vez sin resultados. Al final, al cabo de los años acabaría reconociendo que todo fue un montaje para remontar la crisis que sufría por entonces su empresa de marketing ${ }^{61}$. Lo que ocurrió fue que esta confesión apenas concitó el interés de los medios, por lo que muchos ciudadanos siguieron creyendo que había sido real ${ }^{62}$. Y con similar dureza se criticaron también las teorías de Key, otro de los autores paradigmáticos de esta disciplina ${ }^{63}$.

En un intento por sintetizar la investigación generada en torno a este tema, Pratkanis y Aronson llevaron a cabo en 1992 una exhaustiva revisión bibliográfica sobre los métodos subliminales aplicados a la publicidad para intentar dilucidar su verdadero impacto. Y su conclusión fue que «en ninguno de esos artículos y documentos se aprecian pruebas fehacientes de que los mensajes subliminales influyan en la conducta. Muchos de los estudios no llegan a encontrar un efecto, y los que sí lo encuentran adolecen de diversos fallos metodológicos o no pueden reproducirse ${ }^{64}$. A una postura similar han llegado también otros autores, que han pretendido corroborar así la inexistencia de pruebas empíricas que denoten la existencia de efectos subliminales en el ámbito comercial, tales como provocar conductas específicas o cambios de motivación en los consumidores ${ }^{65}$.

61 WeIR, W. (1958): «Another look at subliminal 'facts'», Advertising Age, 15 de octubre, 46; Durandin, G. (1983): La mentira en la propaganda política y en la publicidad, Barcelona: Paidós; Clark, E. (1989): La publicidad y su poder, Barcelona: Planeta.

62 Pérez Carballada, C. (2009): «Créase o no, la publicidad subliminal no existe». En Marketísimo, 23 de noviembre, http://marketisimo.blogspot.com.es/2009/11/crease-o-no-lapublicidad-subliminal-no.html Web visitada el 19/01/2013.

63 Moore, T.E. (1982): «Subliminal advertising: what you see is what you get», Journal of Marketing, 46 (2), 38-47; Cuperfain, R. y Clarke, T.K. (1985): «A new perspective of subliminal perception», Journal of Advertising, 14 (1), 36-41.

64 Pratkanis, A. y Aronson, E. (1994): La era de la propaganda: uso y abuso de la persuasión, Barcelona: Paidós, 270.

65 George, S.G. y Jenning, L.B. (1975): «Effect of subliminal stimuli on consumer behavior: negative effect», Perceptual and Motor Skills, 41 (3), diciembre, 847-854; Moore, T.E. (1982): «Subliminal advertising: what you see is what you get», Journal of Marketing, 46 (2), 38-47; SAEGERT, J. (1987): «Why marketing should quit giving subliminal advertising the benefit of the doubt», Psychology and Marketing, 4 (2), 107-120; Reingold, E.M. y Merikle, P.M. (1988): «Using direct and indirect measures to study perception without awareness», Perception \& Psychophysics, 44 (6), 563-575; Cuesta, U. (1990): «Efectos de la publicidad subliminal sobre la conducta de intención de compra», Psicología española en la Europa de los 90, vol. 4, Valencia: Psicología del Trabajo y de las Organizaciones, 172-176; BranNon, L.A. y Brock, T.C. (1994): «Test of schema correspondance theory of persuasion: effects of matching an appeal to actual, ideal and product selves», en Clark, E.M.; Brock, T.C. y STEWART, D.W. (eds.) (1994): Attention, attitude, and affect in response to advertising. Hillsdale N.J.: L. Erblaum Associates, 169-188; Theus, K.T. (1994): «Subliminal advertising and the psychology of processing unconscious stimuli: a review research», Psychology \& Marketing, 11, 271-290; TraPPERY, C. (1996): «A meta-analysis of consumer choice and subliminal advertising», Psychology \& Marketing, 13 (5), 517-530; NúÑEz PARTido, J.P. (2000): «Publicidad subliminal. Mito y realidad», Rev. Razón y Fe, 1223-1224, 207-219; Kotler, P. y Armstrong, G. (2003): Fundamentos de marketing, 
Ésta es la opinión más extendida entre la comunidad científica, aunque no se puede pasar por alto que se encuentran también otros trabajos que vienen a incidir en lo contrario, es decir, en que sí existen ciertos efectos ${ }^{66}$. A este respecto no hay que olvidar tampoco que en los últimos años se ha producido un renacimiento del interés acerca del poder del subconsciente humano, coincidiendo con el auge del neuromarketing, disciplina surgida de la unión entre la investigación de los resortes del cerebro humano y el mundo comercial. Los expertos en este ámbito han puesto sobre la mesa que muchas de las decisiones de compra de los consumidores se basan en procesos cerebrales de carácter emocional más que racional que, en muchos casos, operan por debajo del umbral de la conciencia, intentando encontrar por tanto la forma de activar esos resortes para influir sobre los consumidores. Y aunque entendemos que no puede asimilarse el neuromarketing o las neurociencias con lo subliminal, la realidad es que diversos autores, como Lindstrom ${ }^{67}$ y Fisher y Espejo ${ }^{68}$, han venido a afirmar que estos avances están conduciendo en la práctica a que la publicidad subliminal, de una u otra forma, esté hoy más presente que nunca.

\subsection{Investigaciones en el campo de la comunicación politica}

Así como en el ámbito comercial han proliferado las investigaciones sobre el poder de la publicidad subliminal, no ha ocurrido lo mismo en el campo de la comunicación política, donde apenas se encuentran trabajos empíricos. De hecho, muchos de ellos no surgieron hasta que se produjo la polémica por el spot de «RATS».

A este respecto, el consultor Luis Arroyo cree que, en principio, «nada hace suponer que los seres humanos actúen de manera diferente para los spots electorales que para los spots en el resto de los sectores. Se han demostrado ciertos efectos, limitados, pero significativos, en los estímulos subliminales, publicitarios o no. Es por tanto posible que estos estímulos tengan también efectos en la publicidad electoral, aunque tan pequeños que no merezca mucho la pena usarlos».

Madrid: Pearson; Perloff, R.M. (2010): The dynamics of persuasion: communication and attitudes in the 21st. century, Nueva York: Routledge.

66 Bornstein, R.F. y Pittman, T.S. (1992): Perception without awareness: cognitive, clinical, and social perspectives, vol. XII, Nueva York: Guilford Press; GreEnwaLD, A.G. (1992): «New look 3: Unconscious cognition reclaimed», American Psychologist, 47, 766-779; GreENwALD, A.G.; Draine, S.C. y ABrams, R.L. (1996): «Three cognitive markers of unconscious semantic activation», Science, 273, 1699-1702; Dijksterhuis, A.; AArTs, H. y Smith, P.K. (2005): «The power of the subliminal: on subliminal persuasion and other potential applications», en Hassin, R.R.; Uleman, J.S. y Bargh, J.A. (eds.) (2005): The new unconscious, Nueva York: Oxford University Press, 77-106; Claus, J.; Karremans, J.C. y Stroebe, W. (2006): «Beyond Vicary's fantasies: the impact of subliminal priming and brand choice», Journal of Experimental Social Psychology, 42 (6), 792-798; Chartrand, T.L.; Huber, J.; Shiv, B. y Tanner, R.J. (2008): «Nonconscious goals and consumer choice», Journal of Consumer Research, 35, 189-201.

67 Lindstrom, M. (2010): Buyology, verdades y mentiras sobre por qué compramos, Madrid: Gestión 2000.

68 Fisher, L. y Espejo, J. (2011): Mercadotecnia, México: McGraw-Hill. 
Éste es, nuevamente, el centro del debate. Y en este ámbito, se ha suscitado la misma controversia que en el entorno comercial, puesto que los resultados de las investigaciones no son coincidentes. Así, se encuentran por un lado quienes subrayan que los mensajes subliminales no tienen efectos sobre el voto ${ }^{69}$ y quienes, en el lado contrario, consideran que sí pueden influir sobre las actitudes políticas e incluso sobre la intención de voto de los ciudadanos.

Entre estos últimos, han destacado por ejemplo los trabajos de Weinberger y Westen. En un primer experimento, llevado a cabo a través de Internet, proyectaron una fotografía de un candidato anónimo a la que acompañaron unas veces de un mensaje subliminal que ponía «RATS» y otras «STAR» (la misma palabra, pero escrita al revés). Tras la proyección, pidieron a los internautas que indicaran qué les transmitía ese candidato en base a una serie de variables (como honestidad, confianza, competencia...), comprobando que los que habían visto la foto con el mensaje «RATS» valoraron a ese candidato de una forma significativamente más negativa que los que vieron la proyección con la palabra «STAR» $\rangle^{70}$. Estos mismos autores volvieron a repetir su ensayo años después, esta vez con un candidato real (el gobernador de California, Gray Davis), encontrando de nuevo resultados similares ${ }^{71}$. En este caso, insertaron subliminalmente una fotografía del presidente Clinton y apreciaron que esta asociación hizo que los participantes valoraran más positivamente al candidato Davis.

Otro estudio muy similar se llevó a cabo en 2007, cuando un equipo de investigadores de la Universidad Hebrea de Jerusalén realizó un experimento con una muestra de voluntarios en el que comprobaron que la emisión subliminal de imágenes de una bandera de Israel consiguió templar las actitudes más radicales de los participantes e incluso que afectó a su intención de voto ${ }^{72}$. Este mismo equipo avanzó aún más en esta línea de investigación y en 2011 repitió el experimento, pero esta vez con la bandera de Estados Unidos y entre ciudadanos de ese país. En este caso, sus resultados apuntaban que esa imagen condicionaba a los voluntarios hacia actitudes políticas favorables al Partido Republicano y que esa influencia tenía además un efecto prolongado en el tiempo $^{73}$.

\footnotetext{
69 KaID, L.L. (2001): «TechnoDistortions and effects of the 2000 political advertising», American Behavioral Scientist, 44 (12), 2370-2378.

70 Westen, D. (2007): The political brain: the role of emotion in deciding the fate of the nation, Nueva York: Public Affairs Press.

71 Weinberger, J. y Westen, D. (2008): «RATS, we should have used Clinton: subliminal priming in political campaigns», Political Psychology, 29 (5), 631-651.

72 Hassin, R.R.; Ferguson, M.J.; Shidlovski, D. y Gross, T. (2007): «Subliminal exposure to national flags affects political thought and behavior», Proceedings of the National Academy of Science, 104 (50), 19757-19761.

73 Carter, T.J.; Ferguson, M.J. y Hassin, R.R. (2011): «A single exposure to the American flag shifts support toward Republicanism up to 8 months later», Psychological Science, 22 (8), 1.011-1.018.
} 
En esta misma línea, otros autores han llevado a cabo distintos trabajos en los que han incidido en que los mensajes subliminales pueden afectar a la impresión que se tiene de un determinado candidato e influir también sobre cuestiones emocionales que pueden acabar repercutiendo en la intención de voto. En muchos casos se ha aludido al «efecto priming», es decir, que la exposición a estos estímulos subliminales influye en la respuesta que se da a estímulos que se reciben con posterioridad y que, por tanto, predisponen y condicionan al sujeto frente a mensajes que puedan llegarle, por ejemplo, desde la oposición, teniendo así un cierto efecto preventivo ${ }^{74}$. Y ha habido también ensayos en los que se ha subrayado que los mensajes subliminales son más eficaces cuando la información que se transmite en los mismos es negativa ${ }^{75}$. De hecho, todos los ejemplos que se han expuesto hasta el momento han sido precisamente en spots negativos, aunque es verdad que esto puede responder simplemente al hecho de que los anuncios de campaña son cada vez más negativos en todos los países del mundo, una de las consecuencias del denominado proceso de "americanización" de las campañas electorales ${ }^{76}$.

Creemos que esta línea de investigación, muy reciente en el tiempo, necesita aún más desarrollo para corroborar los efectos apuntados. Y es que, como apunta Philip S. Holzman, psicólogo de la Universidad de Harvard, quizá estamos en un punto en el que «debemos estudiar más en profundidad los efectos de los mensajes subliminales, que tantas veces han sido ignorados por muchos científicos» ${ }^{77}$. Y esta necesidad se hace aún más actual por cuanto empiezan a generalizarse también las investigaciones sobre la neuropolítica, que aplica los conocimientos de la neurociencia al campo de las campañas electorales y que, al igual que el neuromarketing apuntado anteriormente, pone el foco en la gran fuerza de los factores emocionales y del subconsciente en

74 Marcus, G.E.; Newman, W.R. y Mackuen, M. (2000): Affective intelligence and political judgment, Chicago: University of Chicago Press; MARcus, G.E. (2002): The sentimental citizen: emotion in democratic politics, University Park, PA: The Pennsylvania State University Press; Dijksterhuis, A.; Aarts, H. y Smith, P.K. (2005): «The power of the subliminal: on subliminal persuasion and other potential applications», en HAssin, R.R.; Uleman, J.S. y BARGH, J.A. (eds.) (2005): The new unconscious, Nueva York: Oxford University Press, 77-106; STEwart, P.A. y SCHUBert, J.N. (2006): «Precognitive prime RATS in a 2000 presidential advertisement», The International Journal of Press/Politics, 11 (4), 103-114; BRADER, T. (2006): Campaigning for hearts and minds: how emotional appeals in political ads work, Chicago: The University of Chicago Press; Stewart, P.A. (2008): «Subliminals in the 2000 presidential election: policy implications of applied neuroscience», Public Integrity, 10 (3), 215-232.

75 Nasrallah, M.; Carmel, D. y Lavie, N. (2009): «Murder, she wrote: enhanced sensitivity to negative word valence», Emotion, 9 (5), 609-618.

76 Rodríguez ANDrÉs, R. (2012): «Los efectos de la americanización de las campañas electorales del mundo», Tribuna Norteamericana, 8, 28-38.

77 Perkins, S. (2003): «Campaign ad may have swayed voters subliminally (Dirty RATS)». En The Free Library, 22 de febrero, http:/www.thefreelibrary.com/Campaign ad may have swayed voters subliminally. (Dirty RATS).-a098977985 Web visitada el 23/02/2014. 
la decisión de voto de los ciudadanos ${ }^{78}$. Y, de hecho, como apunta Antonio Núñez, en los últimos años «el término inconsciente colectivo cada vez se repite más en los informes de los institutos de investigación que hacen los pre-test y los post-test de las campañas, que inciden en que los ciudadanos realizamos cada día miles de operaciones simbólicas por debajo del nivel de conciencia, ya que no podemos procesar toda la información de manera consciente», lo que demuestra que el interés por este asunto está de plena actualidad. Y así, como continúa, «creo que todos los profesionales de la consultoría política, más intuitiva que reflexivamente, contamos con el inconsciente de la ciudadanía para trabajar, aunque nuestro sueño sea que los mensajes que diseñamos afloren a nivel consciente, que se reflexione, medite y debata sobre ellos, y no queden flotando en los pliegues de la inconsciencia o la desmemoria».

En cualquier caso, consideramos que no debería olvidarse una segunda derivada en torno a los efectos de lo subliminal en la política, relacionada con las consecuencias que puede tener desde el punto de vista de la comunicación el hecho de que los medios hagan público que un partido ha empleado estos estímulos.

En este escenario, podría decirse que el primer efecto sería de rechazo, puesto que la opinión pública reaccionaría de forma negativa al descubrir que se ha intentado manipularla. Esto conllevaría un serio riesgo de pérdida reputacional para el partido que lanza el anuncio, al que desde los medios y la oposición se acusaría de manipulación y de vulnerar la legislación en el caso de que esté prohibido su uso. Se reproduce aquí el «efecto boomerang» que ha sido descrito en la investigación sobre los efectos de la publicidad negativa, es decir, que el anuncio puede acabar volviéndose en contra de quien lo ha lanzado. Por todo ello, como afirma Ureña, «los riesgos de utilizar este tipo de publicidad son tan altos que quizá sea uno de los motivos por los que no se use».

A este respecto, Gutiérrez-Rubí intenta poner también en una balanza los «pros» y «contras» del uso de estímulos subliminales en campañas electorales, para acabar llegando a una opinión similar. Para este consultor, «los pros serían incidir con tu mensaje negativo respecto a algún candidato, pero lo veo algo nimio y complicado de conseguir. Creo que tienes más contras: sería visto y difundido como una mala práctica, lo que crearía una mala imagen de tu propio candidato»».

Pero puede darse el caso de que un partido decida asumir este riesgo y lance un anuncio subliminal como parte de una estrategia para ganar presencia mediática o intentar centrar el debate sobre un asunto concreto. Como se ha apuntado anteriormente, este tipo de prácticas, cuando han adquirido relevancia pública, han concitado siempre el interés por parte de los periodistas, influyendo así de forma muy destacada sobre la agenda mediática y, con ello, sobre el debate político y ciudadano. Y en este contexto, y aun contando con el rechazo inicial por parte de la opinión pública, si el anuncio consigue poner sobre la mesa y enfocar el interés de los medios en un asunto especialmente

78 Westen, D. (2007): The political brain: the role of emotion in deciding the fate of the nation, Nueva York: Public Affairs Press; Lakoff, G. (2009): The political mind, Nueva York: Penguin Books. 
sensible, puede que ese debate acabe ocultando el de la posible manipulación, con lo que el factor de rechazo inicial puede mitigarse.

Luis Arroyo es muy gráfico a este respecto: «el truco es conocido. Hago saber que se han utilizado secretas herramientas subliminales en mi anuncio y me lo pasan gratis miles de veces los medios de comunicación, multiplicando la eficacia del mensaje nosubliminal del spot. Y ya de paso también el subliminal, que pasa ahora a ser explícito. Dos por uno».

Esto pudo ocurrir en la campaña del año 2000, cuando el spot de «RATS» contribuyó a que, por encima de la polémica sobre el posible uso de técnicas subliminales, la atención mediática se centrara en los riesgos que los republicanos veían en la política sanitaria de Gore. Esto hizo que este tema, especialmente sensible en Estados Unidos, estuviera varios días en boca de periodistas, políticos y ciudadanos, algo que benefició a la campaña de Bush, puesto que era un asunto especialmente crítico que querían utilizar para restar apoyo al rival. Y además, justo en el momento en el que Gore se había puesto por delante de Bush en los sondeos en una campaña que, como se recordará, fue una de las más igualadas de toda la historia. ¿Era esto lo que buscaba el Partido Republicano? Imposible saberlo a día de hoy, puesto que ninguno de los que participaron en esa campaña se ha pronunciado al respecto, pero el resultado final podría llevar a pensar que quizá fue una acción estratégica con ese objetivo. Y, de hecho, en este contexto resultan especialmente reveladoras las declaraciones que efectuó esos días Greg Stevens, veterano consultor republicano, sobre el creador de este spot: «No hay nada que haga Alex Castellanos que sea un accidente» ${ }^{79}$.

\section{Conclusión}

Adentrarse en el estudio de la publicidad subliminal, tanto en el ámbito comercial como político, no está exento de controversia. Una controversia que, en el caso concreto de las campañas electorales, podría resumirse en tres preguntas principales que resumen lo expuesto en estas páginas y que han servido como objetivos para la presente investigación. En primer lugar, ¿se usa o no se usa la publicidad subliminal en la política, es un mito o una realidad? En este trabajo se han apuntado algunos casos de campañas en las que se han reportado supuestos casos, aun cuando en la mayor parte de ellos no se trataría propiamente de publicidad subliminal sino de otros tipos de publicidad encubierta. A pesar de ello, lo más normal es que candidatos y consultores nieguen el uso de esta técnica. Nadie reconoce haber recurrido a ella, aunque al mismo tiempo nadie niega que en alguna ocasión se haya podido aplicar, lo que contribuye sin duda a aumentar su consideración «mítica».

La segunda cuestión tiene que ver con la eficacia o no de este tipo de publicidad. Durante muchos años la mayoría de la academia ha coincidido en que no se han encontrado evidencias suficientemente fuertes como para dar por probado que la publicidad subliminal afecta a los hábitos de compra o de voto de los ciudadanos,

79 The New York Times, 12/09/2000. 
extremo que en muchos casos ha sido negado. Sin embargo, como hemos apuntado, este tema ha generado mucha polémica y, de hecho, se encuentran también en los últimos años diversos trabajos que apuntan en la dirección contraria, muchos de ellos nacidos al abrigo de los nuevos descubrimientos sobre el funcionamiento del cerebro humano alumbrados desde las neurociencias, el neuromarketing y la neuropolítica, que han vuelto a reabrir el debate sobre la importancia que tiene en nuestro comportamiento la emocionalidad y el subconsciente y que, por tanto, plantean la conveniencia de seguir ahondando en la investigación.

Y, por último, la tercera cuestión tiene que ver con la naturaleza manipuladora de esta técnica. ¿Es ético usar la publicidad subliminal en política? Es este punto el único en el que no parece haber controversia, puesto que se incide en la falta de moralidad del hecho de intentar influir sobre los ciudadanos con técnicas ocultas y sin que, por tanto, sean conscientes de ello. En palabras de Quesnel, la publicidad subliminal anula «toda libertad individual y colectiva» ${ }^{80} \mathrm{y}$, según Becerra, «constituye una práctica muy poco ética ya que coarta la libertad al reducir la posibilidad de elegir» ${ }^{81}$. Y esta opinión es compartida también por los propios consultores políticos, como se ha apuntado. Es precisamente por ello por lo que ha sido prohibida en muchos países y también lo que explica que desde la política se niegue su posible uso y se reniegue de ella, lo que ha llevado igualmente a que este asunto apenas haya concitado la atención de la academia.

Sin embargo, y a modo de cierre, no hay que olvidar que en los últimos años este debate ético se topa con nuevos frentes. Porque si bien hay un rechazo generalizado a la publicidad subliminal, no parece ocurrir lo mismo con los postulados de la neuropolítica, cuando ambas disciplinas están basadas en la apelación al inconsciente humano para influir sobre el comportamiento. Se rechaza, por tanto, una técnica concreta, como es la publicidad subliminal, pero no la filosofía que subyace tras ella, que es lograr la influencia a través del inconsciente. Y así, nos encontramos en la actualidad con la controversia de que mientras que desde distintos ámbitos se afirma que el neuromarketing o la neuropolítica es la publicidad subliminal del siglo XXI, desde otros se rechaza esta asimilación y se considera que, en el fondo, apelar a la emocionalidad y al inconsciente no es sino el reflejo de cómo funciona nuestro cerebro a la hora de tomar decisiones.

El debate, por tanto, está inacabado y la controversia se ha visto alimentada en los últimos años con estas nuevas consideraciones. Y a esto, sin duda, han contribuido decisivamente los medios de comunicación, que han encontrado en este tema una fuente inagotable y muy atractiva de noticias. No hay más que hacer una búsqueda en Internet sobre el concepto de «publicidad subliminal» para darse cuenta de que este asunto, muchas décadas después de su alumbramiento, sigue de permanente actualidad, lo que obliga a los académicos a seguir profundizando en la investigación.

80 Quesnel, L. (1976): «Persuadir en política», en GAUQUELIN, F. (dir.) (1976): El mecanismo de la persuasión, Bilbao: Mensajero, 177-227, 224.

81 Becerra Navarro, A.B. (2005): «El mensaje subliminal: tácticas de publicidad ilícita», Información para la paz, Valencia: Fundación COSO, 169-182, 170. 


\section{Bibliografía}

Acland, C.R. (2011): Swift viewing: the popular life of subliminal influence, Durham, NC: Duke University Press.

BeatTy, S.E. y Hawkins, D.I. (1989): «Subliminal stimulation: some new data and interpretation», Journal of Advertising, 18 (3), 4-8.

Becerra Navarro, A.B. (2005): «El mensaje subliminal: tácticas de publicidad ilícita», Información para la paz, Valencia: Fundación COSO, 169-182.

Belle, N. (2008): «Subliminal message in McCain's new ad?». En Crooks and Liars, 19 de julio, http://crooksandliars.com/2008/07/20/subliminal-message-in-mccainsnew-ad Web visitada el 15/02/2014.

Bevan, W. (1964): «Subliminal stimulation: a pervasive problem for psychology», Psychological Bulletin, 61 (2), 89-99.

BorNSTEIN, R.F. (1989): «Subliminal techniques as propaganda tools: review and critique», The Journal of Mind and Behavior, 10 (3), 231-262.

BornsteIn, R.F. y PitTMan, T.S. (1992): Perception without awareness: cognitive, clinical, and social perspectives, vol. XII, Nueva York: Guilford Press.

BRADER, T. (2006): Campaigning for hearts and minds: how emotional appeals in political ads work, Chicago: The University of Chicago Press.

BrANNON, L.A. y BRock, T.C. (1994): «Test of schema correspondance theory of persuasion: effects of matching an appeal to actual, ideal and product selves», en Clark, E.M.; Brock, T.C. y Stewart, D.W. (eds.) (1994): Attention, attitude, and affect in response to advertising. Hillsdale N.J.: L. Erblaum Associates, 169-188.

Brown, J.A.C. (1986): Técnicas de persuasión: de la propaganda al lavado de cerebro, Madrid: Alianza.

Carter, T.J.; Ferguson, M.J. y Hassin, R.R. (2011): «A single exposure to the American flag shifts support toward Republicanism up to 8 months later», Psychological Science, 22 (8), 1.011-1.018.

Cavazza, N. (1999): Comunicación y persuasión, Madrid: Acento.

Chartrand, T.L.; Huber, J.; Shiv, B. y Tanner, R.J. (2008): «Nonconscious goals and consumer choice», Journal of Consumer Research, 35, 189-201.

Clark, E. (1989): La publicidad y su poder, Barcelona: Planeta.

Claus, J.; Karremans, J.C. y Stroebe, W. (2006): «Beyond Vicary's fantasies: the impact of subliminal priming and brand choice», Journal of Experimental Social Psychology, 42 (6), 792-798.

Cousins, N. (1957): «Smudging the subconscious», Saturday Review, 40, 5 de octubre, 20.

CUESTA, U. (1990): «Efectos de la publicidad subliminal sobre la conducta de intención de compra», Psicología española en la Europa de los 90, vol. 4, Valencia: Psicología del Trabajo y de las Organizaciones, 172-176.

Cuperfain, R. y Clarke, T.K. (1985): «A new perspective of subliminal perception», Journal of Advertising, 14 (1), 36-41.

Disksterhuis, A.; Aarts, H. y Smith, P.K. (2005): «The power of the subliminal: on subliminal persuasion and other potential applications», en HASSIN, R.R.; UlEMAN, J.S. y BARGH, J.A. (eds.) (2005): The new unconscious, Nueva York: Oxford University Press, 77-106. 
Dixon, N.F. (1971): Subliminal perception: the nature of a controversy, Londres: McGraw-Hill.

DurÁn, A. (1989): Psicología de la publicidad y de la venta, Barcelona: CEAC.

Durandin, G. (1983): La mentira en la propaganda política y en la publicidad, Barcelona: Paidós.

Edmonds, T.N. (1988): «Regulate political ads?», Advertising Age, 59 (51), noviembre, $17-20$.

FernÁndez GARRIDO, J. (1987): «Actuaciones publicitarias problemáticas: un estudio de su presencia en los medios de comunicación de masas», Estudios sobre consumo, $11,85-95$.

FERRÉs, J. (1996): Televisión subliminal, Barcelona: Paidós.

Fisher, L. y EsPejo, J. (2011): Mercadotecnia, México: McGraw-Hill.

García Matilla, E. (1979): «Nuevas técnicas de manipular», Mensaje y Medios, 6, enero, 41-45.

(1990): Subliminal: escrito en nuestro cerebro, Madrid: Bitácora.

George, S.G. y Jenning, L.B. (1975): «Effect of subliminal stimuli on consumer behavior: negative effect», Perceptual and Motor Skills, 41 (3), diciembre, 847-854.

Gold, P. (1987): Advertising, politics, and American culture: from salesmanship to therapy, Nueva York: Paragon House.

GonzéLez, J.L. (1994): Persuasión subliminal y sus técnicas, Madrid: Biblioteca Nueva.

Greenwald, A.G. (1992): «New look 3: Unconscious cognition reclaimed», American Psychologist, 47, 766-779.

Greenwald, A.G.; Draine, S.C. y Abrams, R.L. (1996): «Three cognitive markers of unconscious semantic activation», Science, 273, 1699-1702.

HABER, R.N. (1959): «Public attitudes regarding subliminal advertising», Public Opinion Quarterly, 23, verano, 291-293.

Haberstroh, J. (1994): Ice cube sex, Notre Dame: Cross Cultural Publications.

Hassin, R.R.; Ferguson, M.J.; Shidlovski, D. y Gross, T. (2007): «Subliminal exposure to national flags affects political thought and behavior», Proceedings of the National Academy of Science, 104 (50), 19757-19761.

Hawkins, D. (1970): «The effects of subliminal stimulation on drive level and brand preference», Journal of Marketing Research, 7, agosto, 322-326.

Hermida, J.M. (1993): La estrategia de la mentira, Madrid: Temas de Hoy.

JAmieson, K.H. (1992): Dirty politics, Nueva York: Oxford University Press.

KAID, L.L. (2001): «TechnoDistortions and effects of the 2000 political advertising», American Behavioral Scientist, 44 (12), 2370-2378.

Key, W.B. (1991): Seducción subliminal, Buenos Aires: Vergara.

Kotler, P. y Armstrong, G. (2003): Fundamentos de marketing, Madrid: Pearson.

Lakoff, G. (2009): The political mind, Nueva York: Penguin Books.

Lillo, J. (1993): Psicología de la percepción, Madrid: Debate.

LindSTROM, M. (2010): Buyology, verdades y mentiras sobre por qué compramos, Madrid: Gestión 2000.

Marcus, G.E. (2002): The sentimental citizen: emotion in democratic politics, University Park, PA: The Pennsylvania State University Press. 
Marcus, G.E.; Newman, W.R. y Mackuen, M. (2000): Affective intelligence and political judgment, Chicago: University of Chicago Press.

MARK, D. (2006): Going dirty: the art of negative campaigning, Lanham: Rowman \& Littlefield Publishers, Inc.

Méndez, R.M. y Vilalta, A.E. (1999): La publicidad ilícita: engañosa, desleal, subliminal y otras, Barcelona: Bosch.

Merino, D. (1995): Publicidad subliminal y eficacia publicitaria, Madrid: Comunidad de Madrid.

Moix, J. (1997): «Percepción subliminal de estímulos de corta duración», Psicologemas, 11 (22), 189-208.

Moore, T.E. (1982): «Subliminal advertising: what you see is what you get», Journal of Marketing, 46 (2), 38-47.

Morao, J. (2011): «¿Es efectiva la publicidad subliminal?». En Jingle Electoral, 2 de septiembre, http://jingleelectoral.com/2011/09/02/de-la-llamada-publicidadsubliminal/ Web visitada el 14/02/2014.

Muñoz Espinalt, C. (1963): Psicología de la publicidad, Barcelona: Toray.

Nasrallah, M.; Carmel, D. y LaVIE, N. (2009): «Murder, she wrote: enhanced sensitivity to negative word valence», Emotion, 9 (5), 609-618.

NúÑez Partido, J.P. (2000): «Publicidad subliminal. Mito y realidad», Rev. Razón y Fe, 1223-1224, 207-219.

Ogilvy, D. (1985): Ogilvy on advertising, Nueva York: Vintage Books.

O'Toole, J. (1985): The trouble with advertising, Nueva York: Times Books.

PACKARD, V. (1961): Las formas ocultas de la propaganda, Buenos Aires: Editorial Sudamericana.

Pérez Carballada, C. (2009): «Créase o no, la publicidad subliminal no existe». En Marketísimo, 23 de noviembre, http://marketisimo.blogspot.com.es/2009/11/ crease-o-no-la-publicidad-subliminal-no.html Web visitada el 19/01/2013.

PERKINS, S. (2003): «Campaign ad may have swayed voters subliminally (Dirty RATS)». En The Free Library, 22 de febrero, http://www.thefreelibrary.com/Campaign ad may have swayed voters subliminally. (Dirty RATS).-a098977985 Web visitada el 23/02/2014.

Perloff, R.M. (2010): The dynamics of persuasion: communication and attitudes in the 21st. century, Nueva York: Routledge.

PeytiBI, X. (2011): «La mano de Karl Rove es alargada», en el blog Xabier Peytibi, Comunicación política, campañas, redes, 8 de julio, http://www.xavierpeytibi. com/2011/07/08/la-mano-karl-rove-alargada/ Web visitada el 14/03/2014.

PhiLLIPS, M.J. (1997): Ethics and manipulation in advertising, Westport: Quorum Books.

Pinillos, J.L. (1995): Principios de psicología, Madrid: Alianza.

Poetzl, O. (1917): «The relationship between experimentally induced dream images and indirect vision», Psychological Issues, 2, 41-120.

Pratkanis, A. y Aronson, E. (1994): La era de la propaganda: uso y abuso de la persuasión, Barcelona: Paidós.

Qualter, T.H. (1994): Publicidad y democracia en la sociedad de masas, Barcelona: Paidós. 
Quesnel, L. (1976): «Persuadir en política», en Gauquelin, F. (dir.) (1976): El mecanismo de la persuasión, Bilbao: Mensajero, 177-227.

Reingold, E.M. y Merikle, P.M. (1988): «Using direct and indirect measures to study perception without awareness», Perception \& Psychophysics, 44 (6), 563-575.

Rivera Camino, J. y Sutil Martín, L. (2004): Marketing y publicidad subliminal, Madrid: ESIC Editorial.

RodRíGuez ANDRÉs, R. (2012): «Los efectos de la americanización de las campañas electorales del mundo», Tribuna Norteamericana, 8, 28-38.

Rogers, M. y SeILER, C.A. (1994): «The answer is no: a national survey of advertising industry practitioners and their clients about whether they use subliminal advertising», Journal of Advertising Research, 34 (2), 36-45.

SAEGERT, J. (1979): «Another look at subliminal perception», Journal of Advertising Research, 19 (1), 55-57.

(1987): «Why marketing should quit giving subliminal advertising the benefit of the doubt», Psychology and Marketing, 4 (2), 107-120.

SAmuel, L.R. (2013): Freud on Madison Avenue: motivation research and subliminal advertising in America, Philadelphia: University of Pennsylvania Press.

Silverglate, S. (1990): «Subliminal perception and the first amendment: yelling fire in a crowded mind?», University of Miami Law Review, 44, 1243-1281.

SteIN, S. (2008): «Does Romney's ad against McCain contain a subliminal message?». En Huffington Post, 28 de marzo, http://www.huffingtonpost.com/2008/01/04/doesromneys-ad-against-m_n_79905.html Web visitada el 15/02/2014.

STEWART, P.A. (2008): «Subliminals in the 2000 presidential election: policy implications of applied neuroscience», Public Integrity, 10 (3), 215-232.

SteWART, P.A. y SchUbert, J.N. (2006): «Precognitive prime RATS in a 2000 presidential advertisement», The International Journal of Press/Politics, 11 (4), 103-114.

Sutil, L. (1995): Estimulación subliminal: mensajes ocultos que dirigen nuestra vida, Madrid: Vergara.

Theus, K.T. (1994): «Subliminal advertising and the psychology of processing unconscious stimuli: a review research», Psychology \& Marketing, 11, 271-290.

TRAPPERY, C. (1996): «A meta-analysis of consumer choice and subliminal advertising», Psychology \& Marketing, 13 (5), 517-530.

Weinberger, J. y Westen, D. (2008): «RATS, we should have used Clinton: subliminal priming in political campaigns», Political Psychology, 29 (5), 631-651.

WeIR, W. (1958): «Another look at subliminal 'facts'», Advertising Age, 15 de octubre, 46.

Westen, D. (2007): The political brain: the role of emotion in deciding the fate of the nation, Nueva York: Public Affairs Press.

Zanot, E.J.; Pincus, J.D. y Lamp, E.J. (1983): «Public perceptions of subliminal advertising», Journal of Advertising, 12 (1), 39-45. 